\title{
A counterexample to subanalyticity of an arc-analytic function
}

\author{
by KrzyszTof KuRdyKa (Kraków)
}

\begin{abstract}
We construct an arc-analytic function (i.e. a function analytic on each analytic arc) whose graph is not subanalytic.

Let $f: U \rightarrow \mathbb{R}$ be a function, where $U$ is open in $\mathbb{R}^{n}$. We say that $f$ is arc-analytic iff for each analytic arc $\gamma:(-1,1) \rightarrow U$, the composition $f \circ \gamma$ is analytic (see $[\mathrm{K} 2]$, [BM] for examples). If we suppose moreover that $f$ has subanalytic graph it turns out that such an $f$ has some interesting properties. For example if we compose $f$ with a suitable finite composition of local blowing-ups we get an analytic function (see [BM]). In Spring 1985, during the Warsaw Semester on Singularities, after discussions with E. Bierstone, P. Milman and B. Teissier the following conjecture was stated.
\end{abstract}

Conjecture. Every arc-analytic function is locally subanalytic. More precisely, given an arc-analytic function $f: U \rightarrow \mathbb{R}$, where $U$ is open in $\mathbb{R}^{n}$, for each $x \in U$ there is a neighborhood $V_{x}$ of $x$ such that the restriction $f \mid V_{x}$ has subanalytic graph.

In this paper we give a counterexample to this conjecture. The idea of our construction was suggested by an example, due to G. Dloussky, of a mapping which is meromorphic in the sense of Stoll but not in the sense of Remmert (see 5.5 in [D]). I wish to express my gratitude to G. Dloussky for enlightening discussions.

We are going to construct by induction an infinite composition of blowing-ups. Put $X_{0}=\mathbb{R}^{2}, P_{0}=\left\{(x, y) \in \mathbb{R}^{2}: y=0\right\}, c_{0}=(0,0)$. We denote by $\pi_{1,0}: X_{1} \rightarrow X_{0}$ a blowing-up of $X_{0}$ centered at $c_{0}$. Suppose we have already constructed a blowing-up $\pi_{n, n-1}: X_{n} \rightarrow X_{n-1}$ centered at $c_{n-1}$. We denote by $P_{n}$ a strict transform of $P_{n-1}$, and we put $D_{n}=\pi_{n, n-1}^{-1}\left(c_{n-1}\right)$, $\left\{c_{n}\right\}=P_{n} \cap D_{n}$. We take for $\pi_{n+1, n}: X_{n+1} \rightarrow X_{n}$ a blowing-up of $X_{n}$ centered at $c_{n}$. If $n>m$ we put $\pi_{n, m}=\pi_{n, n-1} \circ \ldots \circ \pi_{m+1, m}: X_{n} \rightarrow X_{m}$,

1991 Mathematics Subject Classification: Primary 32B20; Secondary 32B30. 
and $\pi_{n, n}=\operatorname{id}_{X_{n}}$, for $n \in \mathbb{N}$. Clearly $\pi_{n, k}=\pi_{n, m} \circ \pi_{m, k}$ for $n \geq m \geq k$. Hence $\pi_{n, m}: X_{n} \rightarrow X_{m}, n, m \in \mathbb{N}, n \geq m$, is an inverse system. Consider its limit:

$$
\varliminf X_{n}=\left\{\left(x_{n}\right) \in \prod_{n \in \mathbb{N}} X_{n} ; \pi_{n+1, n}\left(x_{n+1}\right)=x_{n}\right\} .
$$

We put $L=\varliminf_{n} \backslash\{c\}$, where $c=\left(c_{n}\right), n \in \mathbb{N}$. Set $p_{k}: L \ni\left(x_{n}\right)_{n \in \mathbb{N}} \mapsto$ $x_{k} \in X_{k}$. We have an induced topology on $X_{n}$, hence also on $L$, such that all $p_{n}, n \in \mathbb{N}$, are continuous. Clearly the topology of $L$ has a countable basis.

Let $x=\left(x_{n}\right)_{n \in \mathbb{N}} \in L$. Then there is an integer $k \in \mathbb{N}$ such that $x_{n} \neq c_{n}$ for all $n \geq k$. Hence there is a neighborhood $U$ of $x$ (in $L$ ) such that $y_{n} \neq c_{n}$ for all $n \geq k$ and all $y=\left(y_{n}\right)_{n \in \mathbb{N}} \in U$.

Notice that $\pi_{n, m} \mid U_{n}: U_{n} \rightarrow U_{m}, n \geq m \geq k$, is an analytic diffeomorphism, where $U_{i}=p_{i}(U), i \in \mathbb{N}$. Thus $p_{n} \mid U: U \rightarrow U_{n}, n \geq k$, is a homeomorphism on $U_{n}$ which is a neighborhood of $x_{n} \in X_{n}$. The family of all such projections defines on $L$ the structure of a real analytic manifold such that all projections $p_{n}: L \rightarrow X_{n}, n \in \mathbb{N}$, are analytic, and moreover each $p_{n}$ has an analytic inverse on $X_{n} \backslash\left\{c_{n}\right\}$.

Consider $p=p_{0}: L \rightarrow \mathbb{R}^{2}$; clearly $p$ has an analytic inverse on $\mathbb{R}^{2} \backslash(0,0)$.

Now take an analytic arc $\gamma:(-1,1) \rightarrow \mathbb{R}^{2}, \gamma(0)=(0,0), \gamma=\left(\gamma_{1}, \gamma_{2}\right)$, and suppose $\gamma_{2}(t) \neq 0$ for $t \neq 0$. Assume that $\gamma_{2}$ has multiplicity $k$ at 0 . The mapping $\widetilde{\gamma}_{n}(t)=\pi_{n, 0}^{-1} \circ \gamma(t)$, for $t \neq 0$, can be extended analytically to 0 . Notice that if $n \geq k$, then $\lim _{t \rightarrow 0} \widetilde{\gamma}_{n}(t) \neq c_{n}$. Hence the arc $\widetilde{\gamma}=p^{-1} \circ \gamma$, for $t \neq 0$, can be extended analytically to 0 , since $p=p_{n} \circ \pi_{n, 0}$.

Let now $\gamma=\left(\gamma_{1}, \gamma_{2}\right)$ be an arc such that $\gamma_{2} \equiv 0$. Then for each compact $K$ in $L$ we can find $\varepsilon>0$ such that $p^{-1} \circ \gamma(t) \notin K$ for all $t$ with $0<|t|<\varepsilon$.

By the Grauert embedding theorem (see $[\mathrm{G}]$ ) the analytic manifold $L$ admits a proper analytic embedding $\alpha: L \rightarrow \mathbb{R}^{N}$, for some $N \in \mathbb{N}$ (by construction the topology of $L$ has a countable basis). Put $G=\alpha \circ p^{-1}$ : $\mathbb{R}^{2} \backslash\{(0,0)\} \rightarrow \mathbb{R}^{N}, G=\left(G_{1}, \ldots, G_{N}\right)$ and $g=\sum_{i=1}^{N} G_{i}^{2}$. By the previous remarks it is obvious that $g$ satisfies the following conditions:

(i) if $\gamma:(-1,1) \rightarrow \mathbb{R}^{2}, \gamma(0)=(0,0)$, is an analytic arc such that $\gamma_{2}(t) \neq 0$ for $t \neq 0$, then the function $g \circ \gamma(t)$, for $t \neq 0$, has an analytic extension to 0 .

(ii) $\lim _{t \rightarrow 0} g(t, 0)=+\infty$.

Finally, we define $f: \mathbb{R}^{2} \rightarrow \mathbb{R}$, putting $f(0,0)=0, f(x, y)=y g(x, y)$ for $(x, y) \neq(0,0)$. By property (i) of $g$ it is clear that $f$ is an arc-analytic function.

Assume now that the restriction $f \mid V$ has subanalytic graph for some neighborhood $V$ of $(0,0)$. Then by the "curve selecting lemma" $f$ is continuous on $V$ (see [K2], [BM]), thus we can assume that $f$ is bounded on $V$. 
Denote by $\tau: \mathbb{R} \rightarrow \mathbb{P}^{1}$ the natural embedding of $\mathbb{R}$ in $\mathbb{P}^{1}, \tau(t)=$ $(t, 1) \in \mathbb{P}^{1}$. Let $\varphi: A \rightarrow \mathbb{R}, A \subset \mathbb{R}^{n}$, be a function. We say that $\varphi$ is subanalytic at infinity $\left(\varphi \in \operatorname{SUBB}\left(\mathbb{R}^{N}\right)\right.$ in the notation of $\left.[\mathrm{K} 1]\right)$ iff the graph of $\tau \circ \varphi$ is subanalytic in $\mathbb{R}^{n} \times \mathbb{P}^{1}$.

Clearly our $f$, being bounded, is subanalytic at infinity; also $h(x, y)=$ $1 / y$, defined for $(x, y) \in \mathbb{R}^{2} \backslash\{y=0\}$, is subanalytic at infinity. Hence the product $g^{\prime}=f \cdot h$ (defined on $V \backslash\{y=0\}$ ) is subanalytic at infinity (cf. [K2]).

Clearly $g^{\prime}=g$ on $V \backslash\{y=0\}$. Since $g$ is continuous on $\mathbb{R}^{2} \backslash\{(0,0)\}$ we have

$$
\lim _{z \rightarrow(0,0)} \sup g(z)=\lim _{z \rightarrow(0,0)} \sup g^{\prime}(z)=+\infty .
$$

From the curve selecting lemma applied to the graph of $\tau \circ g^{\prime}$ at $((0,0), \infty)$ we obtain an analytic arc $\gamma=\left(\gamma_{1}, \gamma_{2}\right), \gamma:(-1,1) \rightarrow \mathbb{R}^{2}$, such that $\gamma(0)=$ $(0,0), \gamma_{2}(t) \neq 0$ for $t \neq 0$ and $\lim _{t \rightarrow 0} g^{\prime} \circ \gamma(t)=\lim _{t \rightarrow 0} g \circ \gamma(t)=\infty$. This gives a contradiction with property (i) of $g$.

In $[\mathrm{BM}]$ Bierstone and Milman asked whether every arc-analytic function is continuous. It is not clear whether or not our function $f$ is continuous.

Addendum. After writing this paper I have learned that an example of an arc-analytic function which is not continuous was constructed by E. Bierstone, P. D. Milman and A. Parusiński in their preprint A function which is arc-analytic but not continuous, Univ. of Toronto, 1990. They also constructed a continuous arc-analytic function whose graph is not subanalytic. Their constructions differ from ours.

\section{References}

[BM] E. Bierstone and P. D. Milman, Arc-analytic functions, preprint, Univ. of Toronto, 1989

[D] G. Dloussky, Analycité séparée et prolongement analytique, preprint, Univ. de Marseille, 1989.

[G] H. Grauert, On Levi's problem and the imbedding of real-analytic manifolds, Ann. of Math. 68 (1958), 460-472.

[K1] K. Kurdyka, Points réguliers d'un sous-analytique, Ann. Inst. Fourier (Grenoble) 38 (1) (1988), 133-156.

[K2] -, Ensembles semi-algébriques symétriques par arcs, Math. Ann. 282 (1988), $445-462$.

INSTITUTE OF MATHEMATICS JAGIELLONIAN UNIVERSITY REYMONTA 4, 30-059 KRAKÓW POLAND 